\title{
BIHARMONIC PROBLEM IN THE HALF-SPACE WITH TRACES IN WEIGHTED SOBOLEV SPACES
}

\author{
CHÉRIF AMROUCHE and YVES RAUDIN
}

(26th June 2006)

\begin{abstract}
In this paper, we study the biharmonic equation in the half-space $\mathbb{R}_{+}^{N}$, with $N \geq 2$. We prove in $L^{p}$ theory, with $1<p<\infty$, existence, uniqueness and regularity results; then we are interested in singular boundary conditions. We consider data and give solutions which live in weighted Sobolev spaces.

Keywords and phrases: Biharmonic problem, Half-space, Weighted Sobolev spaces.
\end{abstract}

\section{Introduction}

The purpose of this paper is the resolution of the biharmonic problem with nonhomogeneous boundary conditions in the half-space

$$
(\mathcal{P}): \quad \Delta^{2} u=f \text { in } \mathbb{R}_{+}^{N}, \quad u=g_{0} \text { and } \partial_{N} u=g_{1} \text { on } \Gamma .
$$

Since this problem is posed in the half-space, it is important to specify the behaviour at infinity for the data and solutions. We have chosen to impose such conditions by setting our problem in weighted Sobolev spaces, where the growth or decay of functions at infinity are expressed by means of weights. These weighted Sobolev spaces provide a correct functional setting for unbounded domains, in particular because the functions in these spaces satisfy an optimal weighted Poincaré-type inequality. Our analysis is based on the isomorphism properties of the biharmonic operator in the whole space and the resolution of the Dirichlet and Neumann problems for the Laplacian in the half-space. This last one is itself based on the isomorphism properties of the Laplace operator in the whole space and also on the reflection principle inherent in the half-space. Note here the double difficulty arising from the

(c) XXXX Australian Mathematical Society 0263-6115/XX $\$ A 2.00+0.00$ 
unboundedness of the domain in any direction and from the unboundedness of the boundary itself.

We begin to establish the existence of generalized solutions to problem $(\mathcal{P})$, i.e. solutions which belong to weighted Sobolev spaces of type $W_{\ell}^{2, p}\left(\mathbb{R}_{+}^{N}\right)$. Then we are interested both in the existence of strong solutions which belong to spaces of type $W_{\ell+2}^{4, p}\left(\mathbb{R}_{+}^{N}\right)$, and singular solutions which belong to $W_{\ell-2}^{0, p}\left(\mathbb{R}_{+}^{N}\right)$ or $W_{\ell-1}^{1, p}\left(\mathbb{R}_{+}^{N}\right)$ in the case $f=0$ with singular boundary conditions. We also establish the existence of solutions which belong to the intermediate spaces $W_{\ell+1}^{3, p}\left(\mathbb{R}_{+}^{N}\right)$.

It turns out that the use of classical Sobolev spaces is inadequate in this case, contrary to the study of elliptic problems of type:

$(\mathcal{Q}): \quad u+\Delta^{2} u=f$ in $\mathbb{R}_{+}^{N}, \quad u=g_{0}$ and $\partial_{N} u=g_{1}$ on $\Gamma$,

where it is more reasonable to consider data and solutions in standard Sobolev spaces. For example, if $f \in L^{2}\left(\mathbb{R}_{+}^{N}\right), g_{0} \in H^{7 / 2}\left(\mathbb{R}^{N-1}\right)$ and $g_{1} \in$ $H^{5 / 2}\left(\mathbb{R}^{N-1}\right)$, problem $(\mathcal{Q})$ admits an unique solution $u \in H^{4}\left(\mathbb{R}_{+}^{N}\right)$. In the case of problem $(\mathcal{P})$, if we assume that $f \in L^{2}\left(\mathbb{R}_{+}^{N}\right)$, the solution $u$ can not be better than in $W_{0}^{4,2}\left(\mathbb{R}_{+}^{N}\right)$ and its traces $\left.u\right|_{\Gamma}$ and $\left.\partial_{N} u\right|_{\Gamma}$ respectively in $W_{0}^{7 / 2,2}\left(\mathbb{R}^{N-1}\right)$ and $W_{0}^{5 / 2,2}\left(\mathbb{R}^{N-1}\right)$. Moreover, we can observe that these spaces are respective subspaces of the first.

On the one hand, we can find in the literature an approach via homogeneous spaces. For instance, when $f \in L^{2}\left(\mathbb{R}_{+}^{N}\right)$, that consists in finding solutions to $(\mathcal{P})$ satisfying $\nabla^{4} u \in L^{2}\left(\mathbb{R}_{+}^{N}\right)^{N^{4}}$, but that gives no information on the other derivatives, nor specifes the behavior at infinity for the data and solutions.

On the other hand, Boulmezaoud has established ( $c f$. [6]) in a Hilbertian framework, the existence of solutions $u \in W_{\ell+1}^{3,2}\left(\mathbb{R}_{+}^{N}\right)$ for data $f \in$ $W_{\ell+1}^{-1,2}\left(\mathbb{R}_{+}^{N}\right)$ and regularity results. However, owing to some critical cases, this framework excludes in particular the dimensions 2 and 4.

To reduce the set of critical values, we have used a special class of weighted Sobolev spaces with logarithmic factors ( $c f$. [2]).

\section{Notations and functional framework}

For any integer $N \geq 2$, writing a typical point $x=\left(x_{1}, \ldots, x_{N}\right) \in \mathbb{R}^{N}$ as $x=\left(x^{\prime}, x_{N}\right)$, we denote by $\mathbb{R}_{+}^{N}=\left\{x \in \mathbb{R}^{N} ; x_{N}>0\right\}$ the upper half-space of $\mathbb{R}^{N}$ and $\Gamma=\left\{\left(x^{\prime}, 0\right) ; x^{\prime} \in \mathbb{R}^{N-1}\right\} \equiv \mathbb{R}^{N-1}$ its boundary.

We shall use the two basic weights $\varrho=\left(1+|x|^{2}\right)^{1 / 2}$ and $\lg \varrho=\ln \left(2+|x|^{2}\right)$, where $|x|=\left(x_{1}^{2}+\cdots+x_{N}^{2}\right)^{1 / 2}$ is the Euclidean norm of $x$.

Let $\lambda=\left(\lambda_{1}, \ldots, \lambda_{N}\right) \in \mathbb{N}^{N}$ be a multi-index and $\partial_{i}=\partial / \partial x_{i}$, then $\partial^{\lambda}=\partial_{1}^{\lambda_{1}} \cdots \partial_{N}^{\lambda_{N}}$ denotes a differential operator of order $|\lambda|=\lambda_{1}+\cdots+\lambda_{N}$. 
For any integer $q, \mathscr{P}_{q}$ stands for the space of polynomials of degree smaller than or equal to $q ; \mathscr{P}_{q}^{\Delta}$ (resp. $\mathscr{P}_{q}^{\Delta^{2}}$ ) is the subspace of harmonic (resp. biharmonic) polynomials of $\mathscr{P}_{q} ; \mathscr{A}_{q}^{\Delta}$ (resp. $\mathscr{N}_{q}^{\Delta}$ ) is the subspace of polynomials of $\mathscr{P}_{q}^{\Delta}$, odd (resp. even) with respect to $x_{N}$, or equivalently, which satisfy the condition $\varphi\left(x^{\prime}, 0\right)=0\left(\operatorname{resp} . \partial_{N} \varphi\left(x^{\prime}, 0\right)=0\right)$; with the convention that these spaces are reduced to $\{0\}$ if $q<0$.

For any real number $s$, we denote by $[s]$ the integer part of $s$.

Given a Banach space $B$, with dual space $B^{\prime}$ and a closed subspace $X$ of $B$, we denote by $B^{\prime} \perp X$ the subspace of $B^{\prime}$ orthogonal to $X$, i.e.

$$
B^{\prime} \perp X=\left\{f \in B^{\prime} ; \forall v \in X,\langle f, v\rangle=0\right\}=(B / X)^{\prime} .
$$

For any $k \in \mathbb{Z}$, we shall denote by $\{1, \ldots, k\}$ the set of the first $k$ positive integers, with the convention that this set is empty if $k$ is nonpositive.

For any real number $p>1$, we always take $p^{\prime}$ to be the Hölder conjugate of $p$, i.e. $1 / p+1 / p^{\prime}=1$.

Let $\Omega$ be an open set of $\mathbb{R}^{N}$. For any $\left.m \in \mathbb{N}, p \in\right] 1, \infty\left[,(\alpha, \beta) \in \mathbb{R}^{2}\right.$, we define the following space:

$$
\begin{aligned}
W_{\alpha, \beta}^{m, p}(\Omega)= & \left\{u \in \mathscr{D}^{\prime}(\Omega) ; 0 \leq|\lambda| \leq k, \varrho^{\alpha-m+|\lambda|}(\lg \varrho)^{\beta-1} \partial^{\lambda} u \in L^{p}(\Omega) ;\right. \\
& \left.k+1 \leq|\lambda| \leq m, \varrho^{\alpha-m+|\lambda|}(\lg \varrho)^{\beta} \partial^{\lambda} u \in L^{p}(\Omega)\right\},
\end{aligned}
$$

where $k=m-\frac{N}{p}-\alpha$ if $N / p+\alpha \in\{1, \ldots, m\}$, and $k=-1$ otherwise.

In the case $\beta=0$, we simply denote the space by $W_{\alpha}^{m, p}(\Omega)$. Note that $W_{\alpha, \beta}^{m, p}(\Omega)$ is a reflexive Banach space equipped with its natural norm:

$$
\begin{aligned}
\|u\|_{W_{\alpha, \beta}^{m, p}(\Omega)}=\left(\sum_{0 \leq|\lambda| \leq k}\left\|\varrho^{\alpha-m+|\lambda|}(\lg \varrho)^{\beta-1} \partial^{\lambda} u\right\|_{L^{p}(\Omega)}^{p}\right. & \\
& \left.\quad \sum_{k+1 \leq|\lambda| \leq m}\left\|\varrho^{\alpha-m+|\lambda|}(\lg \varrho)^{\beta} \partial^{\lambda} u\right\|_{L^{p}(\Omega)}^{p}\right)^{1 / p} .
\end{aligned}
$$

We also define the semi-norm:

$$
|u|_{W_{\alpha, \beta}^{m, p}(\Omega)}=\left(\sum_{|\lambda|=m}\left\|\varrho^{\alpha}(\lg \varrho)^{\beta} \partial^{\lambda} u\right\|_{L^{p}(\Omega)}^{p}\right)^{1 / p} .
$$

The weights in the definition (1) are chosen so that the corresponding space satisfies two properties. On the one hand, $\mathscr{D}\left(\overline{\mathbb{R}_{+}^{N}}\right)$ is dense in $W_{\alpha, \beta}^{m, p}\left(\mathbb{R}_{+}^{N}\right)$. On the other hand, the following Poincaré-type inequality holds in $W_{\alpha, \beta}^{m, p}\left(\mathbb{R}_{+}^{N}\right)$ (cf. [4]):

$$
\text { if } \frac{N}{p}+\alpha \notin\{1, \ldots, m\} \text { or }(\beta-1) p \neq-1,
$$


then the semi-norm $|\cdot|_{W_{\alpha, \beta}^{m, p}\left(\mathbb{R}_{+}^{N}\right)}$ defines on $W_{\alpha, \beta}^{m, p}\left(\mathbb{R}_{+}^{N}\right) / \mathscr{P}_{q^{\prime}}$ a norm which is equivalent to the quotient norm,

$$
\forall u \in W_{\alpha, \beta}^{m, p}\left(\mathbb{R}_{+}^{N}\right), \quad\|u\|_{W_{\alpha, \beta}^{m, p}\left(\mathbb{R}_{+}^{N}\right) / \mathscr{P}_{q^{\prime}}} \leq C|u|_{W_{\alpha, \beta}^{m, p}\left(\mathbb{R}_{+}^{N}\right)},
$$

with $q^{\prime}=\inf (q, m-1)$, where $q$ is the highest degree of the polynomials contained in $W_{\alpha, \beta}^{m, p}\left(\mathbb{R}_{+}^{N}\right)$.

Now, we define the space $\stackrel{\circ}{W}_{\alpha, \beta}^{m, p}\left(\mathbb{R}_{+}^{N}\right)=\overline{\mathscr{D}\left(\mathbb{R}_{+}^{N}\right)}\|\cdot\|_{W_{\alpha, \beta}^{m, p}\left(\mathbb{R}_{+}^{N}\right)}$, and the dual space of $\stackrel{\circ}{W}_{\alpha, \beta}^{m, p}\left(\mathbb{R}_{+}^{N}\right)$ is denoted by $W_{-\alpha,-\beta}^{-m, p^{\prime}}\left(\mathbb{R}_{+}^{N}\right)$. Under the assumption (2), the semi-norm $|\cdot|_{W_{\alpha, \beta}^{m, p}\left(\mathbb{R}_{+}^{N}\right)}$ is a norm on $\stackrel{\circ}{W}_{\alpha, \beta}^{m, p}\left(\mathbb{R}_{+}^{N}\right)$ which is equivalent to the full norm $\|\cdot\|_{W_{\alpha, \beta}^{m, p}\left(\mathbb{R}_{+}^{N}\right)}$.

We shall now recall some properties of the weighted Sobolev spaces $W_{\alpha, \beta}^{m, p}\left(\mathbb{R}_{+}^{N}\right)$. We have the algebraic and topological imbeddings:

$W_{\alpha, \beta}^{m, p}\left(\mathbb{R}_{+}^{N}\right) \hookrightarrow W_{\alpha-1, \beta}^{m-1, p}\left(\mathbb{R}_{+}^{N}\right) \hookrightarrow \cdots \hookrightarrow W_{\alpha-m, \beta}^{0, p}\left(\mathbb{R}_{+}^{N}\right)$ if $\frac{N}{p}+\alpha \notin\{1, \ldots, m\}$.

When $\frac{N}{p}+\alpha=j \in\{1, \ldots, m\}$, then we have:

$$
W_{\alpha, \beta}^{m, p} \hookrightarrow \cdots \hookrightarrow W_{\alpha-j+1, \beta}^{m-j+1, p} \hookrightarrow W_{\alpha-j, \beta-1}^{m-j, p} \hookrightarrow \cdots \hookrightarrow W_{\alpha-m, \beta-1}^{0, p} .
$$

Note that in the first case, for any $\gamma \in \mathbb{R}$ such that $\frac{N}{p}+\alpha-\gamma \notin\{1, \ldots, m\}$ and $m \in \mathbb{N}$, the mapping $u \in W_{\alpha, \beta}^{m, p}\left(\mathbb{R}_{+}^{N}\right) \longmapsto \varrho^{\gamma} u \in W_{\alpha-\gamma, \beta}^{m, p}\left(\mathbb{R}_{+}^{N}\right)$ is an isomorphism. In both cases and for any multi-index $\lambda \in \mathbb{N}^{N}$, the mapping $u \in W_{\alpha, \beta}^{m, p}\left(\mathbb{R}_{+}^{N}\right) \longmapsto \partial^{\lambda} u \in W_{\alpha, \beta}^{m-|\lambda|, p}\left(\mathbb{R}_{+}^{N}\right)$ is continuous. Finally, it can be readily checked that the highest degree $q$ of the polynomials contained in $W_{\alpha, \beta}^{m, p}\left(\mathbb{R}_{+}^{N}\right)$ is given by

$$
q= \begin{cases}m-\left(\frac{N}{p}+\alpha\right)-1 & \text { if }\left\{\begin{array}{l}
\frac{N}{p}+\alpha \in\{1, \ldots, m\} \text { and }(\beta-1) p \geq-1 \\
\text { or } \frac{N}{p}+\alpha \in\{j \in \mathbb{Z} ; j \leq 0\} \text { and } \beta p \geq-1,
\end{array}\right. \\
{\left[m-\left(\frac{N}{p}+\alpha\right)\right]} & \text { otherwise. }\end{cases}
$$

In order to define the traces of functions of $W_{\alpha}^{m, p}\left(\mathbb{R}_{+}^{N}\right)$ (here we don't consider the case $\beta \neq 0$ ), for any $\sigma \in] 0,1[$, we introduce the space:

$$
\begin{array}{r}
W_{0}^{\sigma, p}\left(\mathbb{R}^{N}\right)=\left\{u \in \mathscr{D}^{\prime}\left(\mathbb{R}^{N}\right) ; w^{-\sigma} u \in L^{p}\left(\mathbb{R}^{N}\right) \text { and } \forall i=1, \ldots, N,\right. \\
\left.\int_{0}^{+\infty} t^{-1-\sigma p} d t \int_{\mathbb{R}^{N}}\left|u\left(x+t e_{i}\right)-u(x)\right|^{p} d x<\infty\right\},
\end{array}
$$


where $w=\varrho$ if $N / p \neq \sigma$ and $w=\varrho(\lg \varrho)^{1 / \sigma}$ if $N / p=\sigma$, and $e_{1}, \ldots, e_{N}$ is the canonical basis of $\mathbb{R}^{N}$. It is a reflexive Banach space equipped with its natural norm. Similarly, for any real number $\alpha \in \mathbb{R}$, we define the space:

$$
\begin{aligned}
W_{\alpha}^{\sigma, p}\left(\mathbb{R}^{N}\right)=\left\{u \in \mathscr{D}^{\prime}\left(\mathbb{R}^{N}\right) ; w^{\alpha-\sigma} u \in L^{p}\left(\mathbb{R}^{N}\right),\right. & \\
& \left.\int_{\mathbb{R}^{N} \times \mathbb{R}^{N}} \frac{\left|\varrho^{\alpha}(x) u(x)-\varrho^{\alpha}(y) u(y)\right|^{p}}{|x-y|^{N+\sigma p}} d x d y<\infty\right\},
\end{aligned}
$$

where $w=\varrho$ if $N / p+\alpha \neq \sigma$ and $w=\varrho(\lg \varrho)^{1 /(\sigma-\alpha)}$ if $N / p+\alpha=\sigma$. For any $s \in \mathbb{R}^{+}$, we set

$$
\begin{gathered}
W_{\alpha}^{s, p}\left(\mathbb{R}^{N}\right)=\left\{u \in \mathscr{D}^{\prime}\left(\mathbb{R}^{N}\right) ; 0 \leq|\lambda| \leq k, \varrho^{\alpha-s+|\lambda|}(\lg \varrho)^{-1} \partial^{\lambda} u \in L^{p}\left(\mathbb{R}^{N}\right)\right. \\
\left.k+1 \leq|\lambda| \leq[s]-1, \varrho^{\alpha-s+|\lambda|} \partial^{\lambda} u \in L^{p}\left(\mathbb{R}^{N}\right) ; \partial^{[s]} u \in W_{\alpha}^{\sigma, p}\left(\mathbb{R}^{N}\right)\right\}
\end{gathered}
$$

where $k=s-N / p-\alpha$ if $N / p+\alpha \in\{\sigma, \ldots, \sigma+[s]\}$, with $\sigma=s-[s]$ and $k=-1$ otherwise. We also define, for any real number $\beta$, the space:

$$
W_{\alpha, \beta}^{s, p}\left(\mathbb{R}^{N}\right)=\left\{v \in \mathscr{D}^{\prime}\left(\mathbb{R}^{N}\right) ;(\lg \varrho)^{\beta} v \in W_{\alpha}^{s, p}\left(\mathbb{R}^{N}\right)\right\}
$$

If $u$ is a function on $\mathbb{R}_{+}^{N}$, we denote its trace of order $j$ on the hyperplane $\Gamma$ by:

$$
\forall j \in \mathbb{N}, \quad \gamma_{j} u: x^{\prime} \in \mathbb{R}^{N-1} \longmapsto \partial_{N}^{j} u\left(x^{\prime}, 0\right) .
$$

Let's recall the following trace lemma due to Hanouzet ( $c f$. [8]) and extended by Amrouche-Nečasová ( $c f$. [4]) to this class of weighted Sobolev spaces:

LEMma 2.1. For any integer $m \geq 1$ and real number $\alpha$, the mapping

$$
\gamma=\left(\gamma_{0}, \gamma_{1}, \ldots, \gamma_{m-1}\right): \mathscr{D}\left(\overline{\mathbb{R}_{+}^{N}}\right) \longrightarrow \prod_{j=0}^{m-1} \mathscr{D}\left(\mathbb{R}^{N-1}\right)
$$

can be extended to a linear continuous mapping, still denoted by $\gamma$,

$$
\gamma: W_{\alpha}^{m, p}\left(\mathbb{R}_{+}^{N}\right) \longrightarrow \prod_{j=0}^{m-1} W_{\alpha}^{m-j-1 / p, p}\left(\mathbb{R}^{N-1}\right) .
$$

Moreover $\gamma$ is surjective and $\operatorname{Ker} \gamma=\stackrel{\circ}{W}={ }_{\alpha}^{m, p}\left(\mathbb{R}_{+}^{N}\right)$. 


\section{Biharmonic operator in $\mathbb{R}^{N}$}

Let $\ell \in \mathbb{Z}$. Combining isomorphism results for $\Delta$ in $\mathbb{R}^{N}$ (cf. [2] and [3]), we can show that the following operators are isomorphisms

$$
\begin{aligned}
& \Delta^{2}: W_{\ell}^{2, p}\left(\mathbb{R}^{N}\right) / \mathscr{P}_{[2-\ell-N / p]}^{\Delta^{2}} \longrightarrow W_{\ell}^{-2, p}\left(\mathbb{R}^{N}\right) \perp \mathscr{P}_{\left[2+\ell-N / p^{\prime}\right]}^{\Delta^{2}}, \\
& \Delta^{2}: W_{\ell+1}^{3, p}\left(\mathbb{R}^{N}\right) / \mathscr{P}_{[2-\ell-N / p]}^{\Delta^{2}} \longrightarrow W_{\ell+1}^{-1, p}\left(\mathbb{R}^{N}\right) \perp \mathscr{P}_{\left[2+\ell-N / p^{\prime}\right]}^{\Delta^{2}}, \\
& \Delta^{2}: W_{\ell+2}^{4, p}\left(\mathbb{R}^{N}\right) / \mathscr{P}_{[2-\ell-N / p]}^{\Delta^{2}} \longrightarrow W_{\ell+2}^{0, p}\left(\mathbb{R}^{N}\right) \perp \mathscr{P}_{\left[2+\ell-N / p^{\prime}\right]}^{\Delta^{2}},
\end{aligned}
$$

respectively under the following hypotheses

$$
\begin{aligned}
& \frac{N}{p^{\prime}} \notin\{1, \ldots, \ell\} \quad \text { and } \quad \frac{N}{p} \notin\{1, \ldots,-\ell\}, \\
& \frac{N}{p^{\prime}} \notin\{1, \ldots, \ell+1\} \quad \text { and } \quad \frac{N}{p} \notin\{1, \ldots,-\ell-1\} \text {, } \\
& \frac{N}{p^{\prime}} \notin\{1, \ldots, \ell+2\} \quad \text { and } \quad \frac{N}{p} \notin\{1, \ldots,-\ell-2\} \text {. }
\end{aligned}
$$

\section{Generalized solutions of $\Delta^{2}$ in $\mathbb{R}_{+}^{N}$}

For any $q \in \mathbb{Z}$, we introduce the space $\mathscr{B}_{q}$ as a subspace of $\mathscr{P}_{q}^{\Delta^{2}}$ :

$$
\mathscr{B}_{q}=\left\{u \in \mathscr{P}_{q}^{\Delta^{2}} ; u=\partial_{N} u=0 \text { on } \Gamma\right\} .
$$

THEOREM 4.1 (Generalized solutions). Let $\ell \in \mathbb{Z}$. Under hypothesis (8), for any $f \in W_{\ell}^{-2, p}\left(\mathbb{R}_{+}^{N}\right), g_{0} \in W_{\ell}^{2-1 / p, p}(\Gamma)$ and $g_{1} \in W_{\ell}^{1-1 / p, p}(\Gamma)$ satisfying the compatibility condition

$$
\begin{aligned}
\forall \varphi \in \mathscr{B}_{\left[2+\ell-N / p^{\prime}\right]}, & \\
& \langle f, \varphi\rangle_{W_{\ell}^{-2, p}\left(\mathbb{R}_{+}^{N}\right) \times \stackrel{\circ}{W}_{-\ell}^{2, p^{\prime}}\left(\mathbb{R}_{+}^{N}\right)}+\left\langle g_{1}, \Delta \varphi\right\rangle_{\Gamma}-\left\langle g_{0}, \partial_{N} \Delta \varphi\right\rangle_{\Gamma}=0,
\end{aligned}
$$

problem $(\mathcal{P})$ admits a solution $u \in W_{\ell}^{2, p}\left(\mathbb{R}_{+}^{N}\right)$, unique up to an element of $\mathscr{B}_{[2-\ell-N / p]}$, and there exists a constant $C$ such that

$$
\begin{aligned}
& \inf _{q \in \mathscr{B}_{[2-\ell-N / p]}}\|u+q\|_{W_{\ell}^{2, p}\left(\mathbb{R}_{+}^{N}\right)} \leq \\
& C\left(\|f\|_{W_{\ell}^{-2, p}\left(\mathbb{R}_{+}^{N}\right)}+\left\|g_{0}\right\|_{W_{\ell}^{2-1 / p, p}(\Gamma)}+\left\|g_{1}\right\|_{W_{\ell}^{1-1 / p, p}(\Gamma)}\right) .
\end{aligned}
$$

Proof. 1) We characterize the kernel $\mathscr{K}$ of the operator $\left(\Delta^{2}, \gamma_{0}, \gamma_{1}\right)$ in $W_{\ell}^{2, p}\left(\mathbb{R}_{+}^{N}\right)$. Thanks to the reflection principle for the biharmonic equation (cf. Farwig [7]), we show that if $\ell \in \mathbb{Z}$, assuming that $N / p \notin\{1, \ldots,-\ell\}$, 
then $\mathscr{K}=\mathscr{B}_{[2-\ell-N / p]}$. Moreover, we define the two operators $\Pi_{D}$ and $\Pi_{N}$ respectively for any $r \in \mathscr{A}_{k}^{\Delta}$ and $s \in \mathscr{N}_{k}^{\Delta}$, by:

$$
\Pi_{D} r(x)=\frac{1}{2} \int_{0}^{x_{N}} t r\left(x^{\prime}, t\right) d t \text { and } \Pi_{N} s(x)=\frac{1}{2} x_{N} \int_{0}^{x_{N}} s\left(x^{\prime}, t\right) d t,
$$

satisfying the following properties:

$$
\begin{array}{lll}
\forall r \in \mathscr{A}_{k}^{\Delta}, & \Delta \Pi_{D} r=r \text { in } \mathbb{R}_{+}^{N}, & \Pi_{D} r=\partial_{N} \Pi_{D} r=0 \text { on } \Gamma, \\
\forall s \in \mathscr{N}_{k}^{\Delta}, & \Delta \Pi_{N} s=s \text { in } \mathbb{R}_{+}^{N}, & \Pi_{N} s=\partial_{N} \Pi_{N} s=0 \text { on } \Gamma .
\end{array}
$$

Then we otain a second characterization of the kernel $\mathscr{K}$ which links it to the kernels of the Dirichlet problem and the Neumann problem for the Laplacian in the half-space (cf. [4] and [5]): let $\ell \in \mathbb{Z}$ and assume that $N / p \notin\{1, \ldots,-\ell\}$, then

$$
\mathscr{K}=\mathscr{B}_{[2-\ell-N / p]}=\Pi_{D} \mathscr{A}_{[-\ell-N / p]}^{\Delta} \oplus \Pi_{N} \mathscr{N}_{[-\ell-N / p]}^{\Delta} .
$$

Note that $\mathscr{B}_{[2-\ell-N / p]}=\{0\}$ if $-\ell-N / p<0$.

Using a Green formula and the density of $\mathscr{D}\left(\overline{\mathbb{R}_{+}^{N}}\right)$ in $W_{\ell}^{2, p}\left(\mathbb{R}_{+}^{N}\right)$, we can easily prove the necessity of the compatibility condition (11).

2) We establish the result in the case $f=0$, i.e. for the homogeneous problem:

$$
\left(\mathcal{P}^{0}\right): \quad \Delta^{2} u=0 \text { in } \mathbb{R}_{+}^{N}, \quad u=g_{0} \text { and } \partial_{N} u=g_{1} \text { on } \Gamma .
$$

Thanks to the relation (12), the compatibility condition (11) is equivalent to both conditions:

$$
\forall r \in \mathscr{A}_{\left[\ell-N / p^{\prime}\right]}^{\Delta},\left\langle g_{0}, \partial_{N} r\right\rangle_{\Gamma}=0 \text { and } \forall s \in \mathscr{N}_{\left[\ell-N / p^{\prime}\right]}^{\Delta},\left\langle g_{1}, s\right\rangle_{\Gamma}=0 .
$$

These conditions assure the existence (cf. [4] and [5]) of $\vartheta \in W_{\ell}^{2, p}\left(\mathbb{R}_{+}^{N}\right)$ and $\zeta \in W_{\ell}^{2, p}\left(\mathbb{R}_{+}^{N}\right)$, respectively solutions to the problems

$$
\begin{aligned}
& \Delta \vartheta=0 \text { in } \mathbb{R}_{+}^{N}, \quad \vartheta=g_{0} \text { on } \Gamma, \\
& \Delta \zeta=0 \text { in } \mathbb{R}_{+}^{N}, \quad \partial_{N} \zeta=g_{1} \text { on } \Gamma .
\end{aligned}
$$

We can readily verify that the function defined by

$$
u=x_{N} \partial_{N}(\zeta-\vartheta)+\vartheta \in W_{\ell-1}^{1, p}\left(\mathbb{R}_{+}^{N}\right)
$$

verifies problem $(\mathcal{P})$. Furthermore, we remark that $u$ also satisfies

$$
\Delta u=2 \partial_{N}^{2}(\zeta-\vartheta) \text { in } \mathbb{R}_{+}^{N}, \quad u=g_{0} \text { on } \Gamma .
$$

Then, again thanks to [4] and an unicity argument, we can deduce that $u \in W_{\ell}^{2, p}\left(\mathbb{R}_{+}^{N}\right)$. 
3) In the general case, thanks to Lemma 2.1, we consider a lifting function $u_{g} \in W_{\ell}^{2, p}\left(\mathbb{R}_{+}^{N}\right)$ of $\left(g_{0}, g_{1}\right)$, so that to solve $(\mathcal{P})$ is equivalent solving

$$
\left(\mathcal{P}^{\star}\right): \quad \Delta^{2} u=f \text { in } \mathbb{R}_{+}^{N}, \quad u=\partial_{N} u=0 \text { on } \Gamma,
$$

where $f \in W_{\ell}^{-2, p}\left(\mathbb{R}_{+}^{N}\right)$ with the orthogonality condition $f \perp \mathscr{B}_{\left[2+\ell-N / p^{\prime}\right]}$.

Now, thanks to (3), we can write $f=\operatorname{div} \operatorname{div} \mathbb{F}$, where $\mathbb{F} \in W_{\ell}^{0, p}\left(\mathbb{R}_{+}^{N}\right)^{N^{2}}$.

step 1 . Assume that $2+\ell-N / p^{\prime}<0$. Let $\tilde{\mathbb{F}}$ be the extension of $\mathbb{F}$ to $\mathbb{R}^{N}$ by 0 and $\tilde{f}=\operatorname{div} \operatorname{div} \tilde{\mathbb{F}} \in W_{\ell}^{-2, p}\left(\mathbb{R}^{N}\right)$. By isomorphism (5), since here we have $\mathscr{P} \Delta_{\left[2+\ell-N / p^{\prime}\right]}^{2}=\{0\}$, there exists $\tilde{z} \in W_{\ell}^{2, p}\left(\mathbb{R}^{N}\right)$ such that $\tilde{f}=\Delta^{2} \tilde{z}$ in $\mathbb{R}^{N}$. Writing $z=\left.\tilde{z}\right|_{\mathbb{R}_{+}^{N}}$, we have $f=\Delta^{2} z$ in $\mathbb{R}_{+}^{N}$, with $z \in W_{\ell}^{2, p}\left(\mathbb{R}_{+}^{N}\right)$, $\left.z\right|_{\Gamma} \in W_{\ell}^{2-1 / p, p}(\Gamma)$ and $\left.\partial_{N} z\right|_{\Gamma} \in W_{\ell}^{1-1 / p, p}(\Gamma)$. Since $\mathscr{B}_{\left[2+\ell-N / p^{\prime}\right]}=\{0\}$, the compatibility condition vanishes and point 2) yields the existence of a solution $v \in W_{\ell}^{2, p}\left(\mathbb{R}_{+}^{N}\right)$ to the homogeneous problem

$$
\left(\mathcal{P}^{\sharp}\right): \quad \Delta^{2} v=0 \quad \text { in } \mathbb{R}_{+}^{N}, \quad v=z \text { and } \partial_{N} v=\partial_{N} z \quad \text { on } \Gamma .
$$

The function $u=z-v$ answers to problem $\left(\mathcal{P}^{\star}\right)$ in this case.

step 2. Assume that $2-\ell-N / p<0$. We have shown in the case $2+\ell-N / p^{\prime}<0$, that the operator

$$
\Delta^{2}: \stackrel{\circ}{W}_{\ell}^{2, p}\left(\mathbb{R}_{+}^{N}\right) / \mathscr{B}_{[2-\ell-N / p]} \longrightarrow W_{\ell}^{-2, p}\left(\mathbb{R}_{+}^{N}\right)
$$

is an isomorphism. Thus, by duality we can deduce if $2-\ell-N / p<0$, the isomorphism

$$
\Delta^{2}: \stackrel{\circ}{W_{\ell}^{2, p}}\left(\mathbb{R}_{+}^{N}\right) \longrightarrow W_{\ell}^{-2, p}\left(\mathbb{R}_{+}^{N}\right) \perp \mathscr{B}_{\left[2+\ell-N / p^{\prime}\right]} .
$$

step 3. Assume that $2+\ell-N / p^{\prime} \geq 0$ and $2-\ell-N / p \geq 0$, which implies $\ell \in\{-1,0,1\}$. If $\ell \in\{-1,0\}$, we use again the extension $\tilde{\mathbb{F}}$ of $\mathbb{F}$ to $\mathbb{R}^{N}$ by 0 . By isomorphism (7), since here we have $\mathscr{P}_{\left[\ell-N / p^{\prime}\right]}^{\Delta^{2}}=\{0\}$, there exists $\tilde{\mathbb{G}} \in W_{\ell}^{4, p}\left(\mathbb{R}^{N}\right)^{N^{2}}$ such that $\Delta^{2} \tilde{\mathbb{G}}=\tilde{\mathbb{F}}$ in $\mathbb{R}^{N}$. Writing $\mathbb{G}=\left.\tilde{\mathbb{G}}\right|_{\mathbb{R}_{+}^{N}}$, we have $\Delta^{2} \mathbb{G}=\mathbb{F}$ in $\mathbb{R}_{+}^{N}$. Then setting $z=\operatorname{div} \operatorname{div} \mathbb{G}$, we obtain $z \in W_{\ell}^{2, p}\left(\mathbb{R}_{+}^{N}\right)$ such that $\Delta^{2} z=f$ in $\mathbb{R}_{+}^{N}$; moreover we have $\left.z\right|_{\Gamma} \in W_{\ell}^{2-1 / p, p}(\Gamma)$ and $\left.\partial_{N} z\right|_{\Gamma} \in W_{\ell}^{1-1 / p, p}(\Gamma)$. Since $\mathscr{B}_{\left[2+\ell-N / p^{\prime}\right]}=\{0\}$, point 2) yields a solution $v \in W_{\ell}^{2, p}\left(\mathbb{R}_{+}^{N}\right)$ to problem $\left(\mathcal{P}^{\sharp}\right)$. Then the function $u=z-v$ answers to problem $\left(\mathcal{P}^{\star}\right)$ for $\ell \in\{-1,0\}$. Now we can deduce the case $\ell=1$ by duality from $\ell=-1$.

It remains to combine the three steps to obtain the isomorphism

$$
\Delta^{2}: \stackrel{\circ}{W}_{\ell}^{2, p}\left(\mathbb{R}_{+}^{N}\right) / \mathscr{B}_{[2-\ell-N / p]} \longrightarrow W_{\ell}^{-2, p}\left(\mathbb{R}_{+}^{N}\right) \perp \mathscr{B}_{\left[2+\ell-N / p^{\prime}\right]},
$$

for any $\ell \in \mathbb{Z}$ verifying (8). This answers globally to problem $\left(\mathcal{P}^{\star}\right)$ and thus to general problem $(\mathcal{P})$. 


\section{Weak solutions of $\Delta^{2}$ in $\mathbb{R}_{+}^{N}$}

THEOREM 5.1 (Weak solutions). Let $\ell \in \mathbb{Z}$. Under hypothesis (9), for any $f \in W_{\ell+1}^{-1, p}\left(\mathbb{R}_{+}^{N}\right), g_{0} \in W_{\ell+1}^{3-1 / p, p}(\Gamma)$ and $g_{1} \in W_{\ell+1}^{2-1 / p, p}(\Gamma)$ satisfying the compatibility condition (11), problem $(\mathcal{P})$ admits a solution $u \in W_{\ell+1}^{3, p}\left(\mathbb{R}_{+}^{N}\right)$, unique up to an element of $\mathscr{B}_{[2-\ell-N / p]}$, and which continuously depends on the data with respect to the quotient norm.

Proof. Since $N / p^{\prime} \neq \ell+1$, let's remark that we have the imbedding $W_{\ell+1}^{-1, p}\left(\mathbb{R}_{+}^{N}\right) \hookrightarrow W_{\ell}^{-2, p}\left(\mathbb{R}_{+}^{N}\right)$, then we can easily check that the kernel and the compatibility condition are identical to the ones of Theorem 4.1.

1) We consider a lifting function $u_{g} \in W_{\ell}^{3, p}\left(\mathbb{R}_{+}^{N}\right)$ of $\left(g_{0}, g_{1}\right)$, so that to solve $(\mathcal{P})$ is equivalent solving $\left(\mathcal{P}^{\star}\right)$, where $f \in W_{\ell}^{-1, p}\left(\mathbb{R}_{+}^{N}\right)$ with the orthogonality condition $f \perp \mathscr{B}_{\left[2+\ell-N / p^{\prime}\right]}$.

2) Assume that $l \leq-2$. Thanks to (3), we can write $f=\operatorname{div} \boldsymbol{F}$, where $\boldsymbol{F} \in W_{\ell+1}^{0, p}\left(\mathbb{R}_{+}^{N}\right)^{N}$. Let's denote by $\tilde{\boldsymbol{F}} \in W_{\ell+1}^{0, p}\left(\mathbb{R}^{N}\right)^{N}$ the extension by 0 of $\boldsymbol{F}$ to $\mathbb{R}^{N}$ and $\tilde{f}=\operatorname{div} \tilde{\boldsymbol{F}} \in W_{\ell+1}^{-1, p}\left(\mathbb{R}^{N}\right)$. By isomorphism (6), since here we have $\mathscr{P}_{\left[2+\ell-N / p^{\prime}\right]}^{2}=\{0\}$, there exists $\tilde{z} \in W_{\ell+1}^{3, p}\left(\mathbb{R}^{N}\right)$ such that $\tilde{f}=\Delta^{2} \tilde{z}$ in $\mathbb{R}^{N}$. Writing $z=\left.\tilde{z}\right|_{\mathbb{R}_{+}^{N}}$, we have $f=\Delta^{2} z$ in $\mathbb{R}_{+}^{N}$, with $z \in W_{\ell+1}^{3, p}\left(\mathbb{R}_{+}^{N}\right)$, $\left.z\right|_{\Gamma} \in W_{\ell+1}^{3-1 / p, p}(\Gamma)$ and $\left.\partial_{N} z\right|_{\Gamma} \in W_{\ell+1}^{2-1 / p, p}(\Gamma)$. Thanks to [4] and [5], we also can generalize the result about the homogeneous problem $\left(\mathcal{P}^{0}\right)$ in point 2) of the proof of Theorem 4.1. Thus there exists $v \in W_{\ell+1}^{3, p}\left(\mathbb{R}_{+}^{N}\right)$ solution to $\left(\mathcal{P}^{\sharp}\right)$ and the function $u=z-v$ answers to $\left(\mathcal{P}^{\star}\right)$.

3) Assume that $\ell \geq-1$. There exists an unique $\mu \in \mathscr{A}_{\left[\ell+2-N / p^{\prime}\right]}$ such that for any $\mathscr{A}_{\left[\ell+2-N / p^{\prime}\right]}^{\Delta}$,

$$
\langle f, r\rangle_{W_{\ell+1}^{-1, p}\left(\mathbb{R}_{+}^{N}\right) \times \stackrel{\circ}{W}_{-\ell-1}^{1, p^{\prime}}\left(\mathbb{R}_{+}^{N}\right)}=\int_{\Gamma} \varrho^{-2 \ell-1-N / p+N / p^{\prime}} \partial_{N} \mu \partial_{N} r d x^{\prime},
$$

which we can write

$$
\langle f, r\rangle_{W_{\ell+1}^{-1, p}\left(\mathbb{R}_{+}^{N}\right) \times \stackrel{\circ}{W}_{-\ell-1}^{1, p^{\prime}}\left(\mathbb{R}_{+}^{N}\right)}=\left\langle\xi_{0}, \partial_{N} r\right\rangle_{W_{\ell+1}^{1-1 / p, p}(\Gamma) \times W_{-\ell-1}^{-1 / p^{\prime}, p^{\prime}}(\Gamma)},
$$

setting $\xi_{0}=\varrho^{-2 \ell-1-N / p+N / p^{\prime}} \partial_{N} \mu \in W_{\ell+1}^{1-1 / p, p}(\Gamma)$. That is precisely the compatibility condition of the Dirichlet problem ( $c f$. [4])

$$
\Delta \xi=f \text { in } \mathbb{R}_{+}^{N}, \quad \xi=\xi_{0} \text { on } \Gamma,
$$

which admits then a solution $\xi \in W_{\ell+1}^{1, p}\left(\mathbb{R}_{+}^{N}\right)$. Now we use the characterization (12). Since $f \perp \mathscr{B}_{\left[2+\ell-N / p^{\prime}\right]}$, we have

$$
\forall r \in \mathscr{A}_{\left[\ell-N / p^{\prime}\right]}^{\Delta}, \quad\left\langle\Delta \xi, \Pi_{D} r\right\rangle_{W_{\ell+1}^{-1, p}\left(\mathbb{R}_{+}^{N}\right) \times \stackrel{\circ}{W}_{-\ell-1}^{1, p^{\prime}}\left(\mathbb{R}_{+}^{N}\right)}=\left\langle f, \Pi_{D} r\right\rangle=0 .
$$


By a Green formula and the imbedding $W_{\ell+1}^{1, p}\left(\mathbb{R}_{+}^{N}\right) \hookrightarrow W_{\ell-1}^{-1, p}\left(\mathbb{R}_{+}^{N}\right)$, we can deduce that

$$
\forall r \in \mathscr{A}_{\left[\ell-N / p^{\prime}\right]}^{\Delta}, \quad\left\langle\xi, \Delta \Pi_{D} r\right\rangle_{W_{\ell-1}^{-1, p}\left(\mathbb{R}_{+}^{N}\right) \times \stackrel{\circ}{W}_{-\ell+1}^{1, p^{\prime}}\left(\mathbb{R}_{+}^{N}\right)}=0 .
$$

Thus, we have

$$
\forall r \in \mathscr{A}_{\left[\ell-N / p^{\prime}\right]}^{\Delta}, \quad\langle\xi, r\rangle_{W_{\ell-1}^{-1, p}\left(\mathbb{R}_{+}^{N}\right) \times \stackrel{\circ}{W}_{-\ell+1}^{1, p^{\prime}}\left(\mathbb{R}_{+}^{N}\right)}=0,
$$

which is again the compatibility condition for the Dirichlet problem

$$
\Delta \vartheta=\xi \text { in } \mathbb{R}_{+}^{N}, \quad \vartheta=0 \text { on } \Gamma,
$$

which admits then a solution $\vartheta \in W_{\ell+1}^{3, p}\left(\mathbb{R}_{+}^{N}\right)$. Similarly we have

$$
\forall s \in \mathscr{N}_{\left[\ell-N / p^{\prime}\right]}^{\Delta}, \quad\left\langle\Delta \xi, \Pi_{N} s\right\rangle_{W_{\ell+1}^{-1, p}\left(\mathbb{R}_{+}^{N}\right) \times \stackrel{\circ}{W}_{-\ell-1}^{1, p^{\prime}}\left(\mathbb{R}_{+}^{N}\right)}=\left\langle f, \Pi_{N} s\right\rangle=0,
$$

therefore we have

$$
\forall s \in \mathscr{N}_{\left[\ell-N / p^{\prime}\right]}^{\Delta}, \quad\langle\xi, s\rangle_{W_{\ell-1}^{-1, p}\left(\mathbb{R}_{+}^{N}\right) \times \stackrel{\circ}{W}_{-\ell+1}^{1, p^{\prime}}\left(\mathbb{R}_{+}^{N}\right)}=0,
$$

which is the compatibility condition for Neumann problem (cf. [5])

$$
\Delta \zeta=\xi \text { in } \mathbb{R}_{+}^{N}, \quad \partial_{N} \zeta=0 \text { on } \Gamma,
$$

which admits then a solution $\zeta \in W_{\ell+1}^{3, p}\left(\mathbb{R}_{+}^{N}\right)$. The function defined by

$$
u=x_{N} \partial_{N}(\zeta-\vartheta)+\vartheta \in W_{\ell}^{2, p}\left(\mathbb{R}_{+}^{N}\right)
$$

verifies problem $\left(\mathcal{P}^{\star}\right)$. Furthermore, we remark that $u$ also satisfies

$$
\Delta u=2 \partial_{N}^{2}(\zeta-\vartheta)+\xi \text { in } \mathbb{R}_{+}^{N}, \quad u=0 \text { on } \Gamma .
$$

By [4] and an unicity argument, we can deduce that $u \in W_{\ell+1}^{3, p}\left(\mathbb{R}_{+}^{N}\right)$.

\section{Strong solutions of $\Delta^{2}$ in $\mathbb{R}_{+}^{N}$}

Theorem 6.1 (Strong solutions). Let $\ell \in \mathbb{Z}$. Under hypothesis (10), for any $f \in W_{\ell+2}^{0, p}\left(\mathbb{R}_{+}^{N}\right), g_{0} \in W_{\ell+2}^{4-1 / p, p}(\Gamma)$ and $g_{1} \in W_{\ell+2}^{3-1 / p, p}(\Gamma)$ satisfying the compatibility condition (11), problem $(\mathcal{P})$ admits a solution $u \in W_{\ell+2}^{4, p}\left(\mathbb{R}_{+}^{N}\right)$, unique up to an element of $\mathscr{B}_{[2-\ell-N / p]}$, and which continuously depends on the data with respect to the quotient norm.

Proof. In the case $l \leq-2$, the arguments are similar to the ones of Theorem 5.1. If $\ell \geq-1$, since $N / p^{\prime} \neq \ell+2$, we have the imbedding $W_{\ell+2}^{0, p}\left(\mathbb{R}_{+}^{N}\right) \hookrightarrow W_{\ell+1}^{-1, p}\left(\mathbb{R}_{+}^{N}\right)$. Then, thanks to Theorem 5.1, there exists a solution $u \in W_{\ell+1}^{3, p}\left(\mathbb{R}_{+}^{N}\right)$ to problem $\left(\mathcal{P}^{\star}\right)$ and classical regularity arguments allow us to show that $u \in W_{\ell+2}^{4, p}\left(\mathbb{R}_{+}^{N}\right)$. 


\section{Singular boundary conditions}

THEOREM 7.1. Let $l \in \mathbb{Z}$ and assume that

$$
\frac{N}{p^{\prime}} \notin\{1, \ldots, \ell-2\} \quad \text { and } \quad \frac{N}{p} \notin\{1, \ldots,-\ell+2\} .
$$

For any $g_{0} \in W_{\ell-2}^{-1 / p, p}(\Gamma)$ and $g_{1} \in W_{\ell-2}^{-1-1 / p, p}(\Gamma)$ satisfying the compatibility condition

$$
\forall \varphi \in \mathscr{B}_{\left[2+\ell-N / p^{\prime}\right]}, \quad\left\langle g_{1}, \Delta \varphi\right\rangle_{\Gamma}-\left\langle g_{0}, \partial_{N} \Delta \varphi\right\rangle_{\Gamma}=0,
$$

problem $\left(\mathcal{P}^{0}\right)$ has a solution $u \in W_{\ell-2}^{0, p}\left(\mathbb{R}_{+}^{N}\right)$, unique up to an element of $\mathscr{B}_{[2-\ell-N / p]}$, and which continuously depends on the data with respect to the quotient norm.

Proof. 1) Let $l \in \mathbb{Z}$ and $m \geq 2$ be two integers. We introduce the spaces

$$
\begin{aligned}
\stackrel{\star}{W}_{\ell}^{m, p}\left(\mathbb{R}_{+}^{N}\right) & =\left\{u \in W_{\ell}^{m, p}\left(\mathbb{R}_{+}^{N}\right) ; u=\partial_{N} u=0 \text { on } \Gamma\right\}, \\
Y_{\ell, 1}^{p}\left(\mathbb{R}_{+}^{N}\right) & =\left\{v \in W_{\ell-2}^{0, p}\left(\mathbb{R}_{+}^{N}\right) ; \Delta^{2} v \in W_{\ell+2,1}^{0, p}\left(\mathbb{R}_{+}^{N}\right)\right\} .
\end{aligned}
$$

Note that $Y_{\ell, 1}^{p}\left(\mathbb{R}_{+}^{N}\right)$ is a reflexive Banach space equipped with its natural norm:

$$
\|v\|_{Y_{\ell, 1}^{p}\left(\mathbb{R}_{+}^{N}\right)}=\|v\|_{W_{\ell-2}^{0, p}\left(\mathbb{R}_{+}^{N}\right)}+\left\|\Delta^{2} v\right\|_{W_{\ell+2,1}^{0, p}\left(\mathbb{R}_{+}^{N}\right)} .
$$

Then we can show that under hypothesis $(13)$, the space $\mathscr{D}\left(\overline{\mathbb{R}_{+}^{N}}\right)$ is dense in $Y_{\ell, 1}^{p}\left(\mathbb{R}_{+}^{N}\right)$. We deduce that the mapping $\left(\gamma_{0}, \gamma_{1}\right): \mathscr{D}\left(\overline{\mathbb{R}_{+}^{N}}\right) \longrightarrow \mathscr{D}\left(\mathbb{R}^{N-1}\right)^{2}$ can be extended to a linear continuous mapping

$$
\left(\gamma_{0}, \gamma_{1}\right): Y_{\ell, 1}^{p}\left(\mathbb{R}_{+}^{N}\right) \longrightarrow W_{\ell-2}^{-1 / p, p}(\Gamma) \times W_{\ell-2}^{-1-1 / p, p}(\Gamma) ;
$$

and moreover we have for any $v \in Y_{\ell, 1}^{p}\left(\mathbb{R}_{+}^{N}\right)$ and $\varphi \in \stackrel{\star}{W^{4}, p^{\prime}}\left(\mathbb{R}_{+}^{N}\right)$,

$$
\begin{gathered}
\left\langle\Delta^{2} v, \varphi\right\rangle_{W_{\ell+2,1}^{0, p}\left(\mathbb{R}_{+}^{N}\right) \times W_{-\ell-2,-1}^{0, p^{\prime}}\left(\mathbb{R}_{+}^{N}\right)}-\left\langle v, \Delta^{2} \varphi\right\rangle_{W_{\ell-2}^{0, p}\left(\mathbb{R}_{+}^{N}\right) \times W_{-\ell+2}^{0, p^{\prime}\left(\mathbb{R}_{+}^{N}\right)}}= \\
\left\langle v, \partial_{N} \Delta \varphi\right\rangle_{W_{\ell-2}^{-1 / p, p}(\Gamma) \times W_{-\ell+2}^{1 / p, p^{\prime}}(\Gamma)}-\left\langle\partial_{N} v, \Delta \varphi\right\rangle_{W_{\ell-2}^{-1-1 / p, p}(\Gamma) \times W_{-\ell+2}^{1+1 / p, p^{\prime}}(\Gamma)} .
\end{gathered}
$$

2) Let $\mathscr{K}^{b}=\left\{z \in W_{\ell-2}^{0, p}\left(\mathbb{R}_{+}^{N}\right) ; \Delta^{2} z=0\right.$ in $\mathbb{R}_{+}^{N}, z=\partial_{N} z=0$ on $\left.\Gamma\right\}$ be the kernel of this operator. Thanks to the formula (15), we can observe that Problem $\left(\mathcal{P}^{0}\right)$ is equivalent to the formulation:




3) We shall solve problem $(\mathcal{Q})$ by duality. According to Theorem 6.1, problem $\left(\mathcal{P}^{\star}\right)$ admits an unique solution $v \in W_{-\ell+2}^{4, p^{\prime}}\left(\mathbb{R}_{+}^{N}\right) / \mathscr{B}_{\left[2+\ell-N / p^{\prime}\right]}$, under hypothesis (13). Moreover, $v$ satisfies the estimate

$$
\|v\|_{W_{-\ell+2}^{4, p^{\prime}}\left(\mathbb{R}_{+}^{N}\right) / \mathscr{B}_{\left[2+\ell-N / p^{\prime}\right]}} \leq C\|f\|_{W_{-\ell+2}^{0, p^{\prime}}\left(\mathbb{R}_{+}^{N}\right)} .
$$

Consider the linear form $T: f \longmapsto\left\langle g_{1}, \Delta v\right\rangle_{\Gamma}-\left\langle g_{0}, \partial_{N} \Delta v\right\rangle_{\Gamma}$. We can show that it is continuous on $W_{-\ell+2}^{0, p^{\prime}}\left(\mathbb{R}_{+}^{N}\right) \perp \mathscr{B}_{[2-\ell-N / p]}$. Then, according to Riesz representation theorem, there exists an unique $u \in W_{\ell-2}^{0, p}\left(\mathbb{R}_{+}^{N}\right) / \mathscr{B}_{[2-\ell-N / p]}$ such that $T f=\langle u, f\rangle_{W_{\ell-2}^{0, p}\left(\mathbb{R}_{+}^{N}\right) \times W_{-\ell+2}^{0, p^{\prime}}\left(\mathbb{R}_{+}^{N}\right)^{\prime}}$. This means that $u$ is a solution to problem $(\mathcal{Q})$ and $\mathscr{K}^{b}=\mathscr{B}_{[2-\ell-N / p]}$.

We can complete these results by an intermediate case, with a proof similar to the previous one:

THEOREM 7.2. Let $l \in \mathbb{Z}$ and assume that

$$
\frac{N}{p^{\prime}} \notin\{1, \ldots, \ell-1\} \quad \text { and } \quad \frac{N}{p} \notin\{1, \ldots,-\ell+1\} .
$$

For any $g_{0} \in W_{\ell-1}^{1-1 / p, p}(\Gamma)$ and $g_{1} \in W_{\ell-1}^{-1 / p, p}(\Gamma)$ satisfying the compatibility condition (14), problem $\left(\mathcal{P}^{0}\right)$ has a solution $u \in W_{\ell-1}^{1, p}\left(\mathbb{R}_{+}^{N}\right)$, unique up to an element of $\mathscr{B}_{[2-\ell-N / p]}$, and which continuously depends on the data with respect to the quotient norm.

\section{References}

[1] R. A. Adams. Sobolev Spaces. Academic Press, New York, 1975.

[2] C. Amrouche, V. Girault, J. Giroire. Weighted Sobolev spaces for Laplace's equation in $\mathbb{R}^{N}$. J. Math. Pures Appl. 73, 6 (1994), 579-606.

[3] C. Amrouche, V. Girault, J. Giroire. Equation de Poisson et espaces de Sobolev avec poids critiques. Publications du Laboratoire de Mathématiques Appliquées de l'Université de Pau.

[4] C. Amrouche, S. NeČasová. Laplace equation in half space with a nonhomogeneous Dirichlet boundary condition. Mathematica Bohemica 126, 2 (2001), 265-274.

[5] C. Amrouche. The Neumann problem in the half space. C. R. Acad. Sci. Paris, Série I 335 (2002), 151-156.

[6] T. Z. Boulmezaoud. On the Stokes system and the biharmonic equation in the half-space: an approach via weighted Sobolev spaces. Mathematical Methods in the Applied Sciences 25 (2002), 373-398. 
[7] R. FArwig. A Note on the Reflection Principle for the Biharmonic Equation and the Stockes system. Acta applicandae Mathematicae 25 (1994), 41-51.

[8] B. Hanouzet. Espaces de Sobolev avec poids. Application au problème de Dirichlet dans un demi-espace. Rend. Sem. Univ. Padova 46 (1971), 227-272.

Laboratoire de Mathématiques Appliquées

Université de Pau et des Pays de l'Adour

IPRA, Avenue de l'Université

$64000 \mathrm{Pau}$

France

e-mail: cherif .amrouche@univ-pau.fr 